\title{
Correlations of the Maxillary Sinus with the Craniofacial Dimensions in Different Age Groups (A Study on Digital Lateral Cephalometric Radiographs of Males in Mosul City)
}

\begin{abstract}
Aims: This study aimed to detect if there are a correlation between the maxillary sinus measurements ( height and depth) with the cranial and facial structures that probably could estimate a functional matrix rule of the maxillary sinus in the growth of these structure add to estimate the percent of increase from age group to another. Materials and Methods: A digital lateral cephalometric radiographs of 113 males with class I malocclusion from Mosul city were divided into three age groups: 14 patients Preadolescent (6-9 years), 70 patients adolescent (11-15 years) and 29 patients adult (17-42 years) with measurements taken involving the maxillary sinus height and depth, maxillary length, mandibular lengths, anterior facial height(upper and lower parts), posterior facial height (upper and lower part), cranial base dimensions then a correlation have been down between the maxillary sinus height and depth with the related dimensions of the craniofacial complex. Results: The correlation between the MSH and the related craniofacial dimensions in the three age groups showed a significantly positive correlations in nearly all of the measurement specially at adolescences and adults with just two significant positive correlations for MRL and TPFH in preadolescence, on the other hand correlating the MSH with the related measurement in general in single group showed a positive significant correlation for all measurement. While correlating the MSD with the related craniofacial dimensions showed a significantly positive correlation with MBL, TML and Nba in adult. For MBL and TML in preadolescence, but no significant correlation showed in adolescence, with a significant correlation for MSD with nearly all the related craniofacial dimensions in all age group in general. In all measurement the percentage of increase from preadolescence to adolescence more than that from adolescence to adult. Conclusions: we could concluded that in the three age groups the growth of the maxillary sinus probably may influence the related craniofacial structures as a functional matrix role in the growth mechanism.
\end{abstract}

Prof $D r$ Khudair A Al-Jumaili (BDS, CES, DSO); Lect Mustafa M. Hamed (BDS, MSc)

Department of Pedodontics, Orthodontics and Preventive Dentistry, Dentistry College, Mosul University

Key words: Maxillary sinus, craniofacial dimensions, correlations, different age groups. 
1 he maxillary sinus is the first of the paranasal sinuses to develop in human foetal life.

During the fifth foetal month, secondary pneumatization starts as the maxillary sinus grows beyond the nasal capsule into the maxilla. At birth, the sinus is approximately $10 \times 3 \times 4$ $\mathrm{mm}$ in dimension and continues to grow slowly until the age of 7 years when expansion occurs more rapidly until all the permanent teeth have erupted. The average dimensions of the maxillary sinus of the adult are $40 \times 26 \times 28 \mathrm{~mm}$ with an average volume of $15 \mathrm{~mL}$. $^{(1)}$ The maxillary sinus is typically pyramidal in shape with the base of the pyramid forming the lateral nasal wall and the apex extending into the zygoma. ${ }^{(2)}$

Based on the functional matrix concept, scientists developed the hypothesis that soft tissue acting on certain bone pieces determines the process of facial growth. The functional matrix theory states that soft tissues acting on the various bony parts composing the face are the determining factor for its anterior and inferior growth pattern. ${ }^{(3)}$

Functional Matrix Hypothesis (Moss Hypothesis) The functional matrix is primary and the presence, size, shape, spatial position, and growth of any skeletal unit is secondary, compensatory, and mechanically obligated to changes in the size, shape, spatial position of its related functional matrix"ss(4,5). There are two types of the functional matrix: Periosteal matrix (Muscles) and the Capsular matrix (brain, oral cavity, orbital cavity, sinuses....etc). ${ }^{(6)}$

The most fundamental dictum of the functional matrix hypothesis was stated by Moss ${ }^{(7)}$ as follows: The origin of all skeletal units, all of their subsequent changes in size, shape and location and their subsequent maintenance and being, are always, and without exception, secondary, compensatory and mechanical obligatory responses to the morphogenetically and temporally prior and primary alterations of the operational (functional) demands of their specifically related functional matrices. Put more succinctly, "Bones do not grow, they are grown." The basic premise of this theory is that bone and cartilage do not regulate their own growth. This theory proposes that craniofacial bony growth occurs as a reaction to or in response to the functional matrix surrounding it. A functional matrix consists of the cells that comprise muscle, soft tissue, teeth, glands, and nerves, as well as the functioning volumes of various cavities associated with the head.

Ballantyne $^{(8)}$ mentioned only the time for the beginning of development of maxillary sinus, Weiglein ${ }^{(9)}$ described detailed metric analysis of maxillary sinus in humans of postnatal period (0-12 years of age), while Lund ${ }^{(10,11)}$ mentioned early cavitation, rate of growth in anteroposterior diameter, length, height and width of maxillary sinus at birth in human foetus.

There was no previous study demonstrated the association between the maxillary sinus dimensions and the craniofacial dimensions add to percentage of increase from age group to another in an electronic search using Pub med.

The purpose of this study is to investigate the maxillary sinus dimensions (height and depth) in different age groups and the association between the maxillary sinus dimensions and the craniofacial dimensions to detect the possibility of sinus effect on the craniofacial structure add to the detecting the percentage of increase in maxillary sinus dimensions and craniofacial dimensions between age groups.

\section{MATERIALS AND METHODS}

Digital lateral cephalometric radiographs data of 113 males with Class I malocclusion visiting the department of radiology, Dental College, Mosul University, Mosul, were assessed and the criteria of selection were class I molar relation no open bite anteriorly or posteriorly, no deep bite. While 14 males (Preadolescent age group) aged 6 to 9 years; mean age $=7.64$ years, 70 males (Adolescent age group) aged 11 to 15 years; mean age $=12.43$ years, 29 males (Adult age 
group) age 17 to 42 years; mean age $=24.9$ years. All the patients were intended to $\mathrm{x}$ ray department for different reasons, most of them for orthodontic purpose . The digital lateral cephalometric radiographs were made by the use of Planmega Dimax Pro X-ray machine with Dimax classic imaging software. The plastic stopper of the cephalostate containing a measuring strap to define a reference distance to calibrate the linear measurements. The cephalometric exposure values were selected and fixed on the cephalostate depending on the age of the subject.

\section{Dimensional measurements:}

Below is an overview of the measurement of the patient's digital lateral cephalometric radiograph employed for exploration of the maxillary sinus and craniofacial dimensions.

1. Maxillary sinus dimensions: Involve Maxillary sinus height (MSH) which represented by a straight vertical line drown from the deepest point in the concavity of sinus roof to the deepest point in the concavity of sinus floor and Maxillary Sinus Depth (MSD) which represented by a horizontal line drown from the deepest point in the concavity of sinus anterior wall to the deepest point in the concavity of the sinus posterior wall.

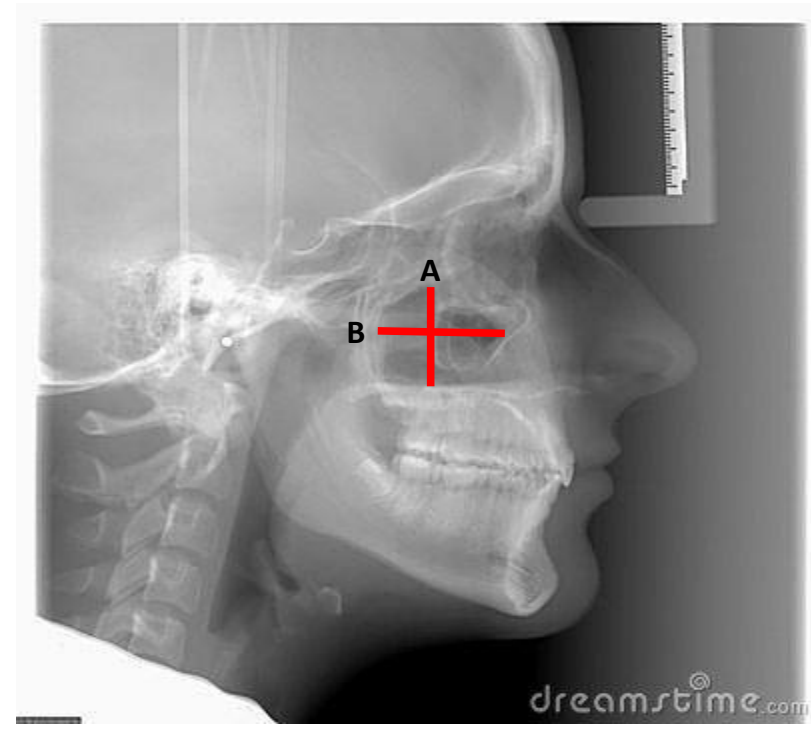

A: represent the Sinus Height

B: represent the Sinus Depth

2. Craniofacial dimensions ${ }^{(12)}$ : Involve anterior cranial base $(\mathrm{SN})$, posterior cranial base (SBa), cranial base length (NBa), upper anterior facial height (UAFH), upper posterior facial height (UPFH), lower anterior facial height (LAFH), lower posterior facial height(LPFH), total anterior facial height (TAFH), total posterior facial height (TPFH), Maxillary length (MaxL), mandibular ramus length (MRL), mandibular body length (MBL) and total mandibular length (TML). 


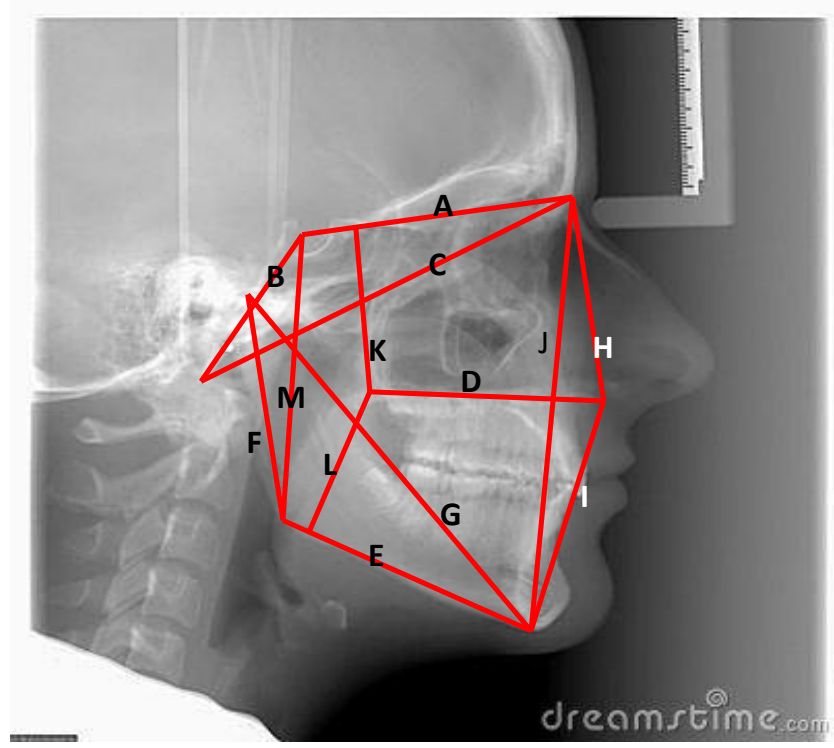

A: represent the anterior cranial base

B: represent the posterior cranial base

C: represent the cranial base depth

D: represent the maxillary length

E: represent the mandibular body length

F: represent the mandibular ramus length

G: represent the total mandibular length

H: represent the upper anterior facial height

I: represent the lower anterior facial height

J: represent the total anterior facial height

K: represent the upper posterior facial height

L: represent the lower posterior facial height

M: represent the total posterior facial height

\section{Statistical analysis:}

A descriptive statistic involving minimum, maximum, mean and standard deviation was done for all measurements with a Pearson correlations between the maxillary sinus dimensions and the craniofacial dimensions involving correlating the MSH with SBa, Nba, UAFH, LAFH, TAFH, TPFH, UPFH, LPFH, MRL and MSD with SN, Nba, MaxL, MBL, TML differences were considered as statistically significant when $p$-values were less than 0.05 . The percentage of increase of each variable from age group to another also assessed.

\section{RESULTS AND DISCUSSION}

The statistical evaluation displayed the data like the group means, minimum, maximum and standard deviations of the all dimensions were listed in Table (1). The correlation between the MSH and the related craniofacial dimensions in the three age groups are presented in Tables $(4,5,6)$ which showed a significantly positive correlations in nearly all of the measurement specially at adolescences and adults with just two significant positive correlations for MRL and TPFH in preadolescence, on the other hand correlating the MSH with the related measurement in general in single group showed a positive significant correlation for all measurement, this is listed in Table (7). While correlating the MSD with the related craniofacial dimensions is listed in Tables $(8,9,10)$ which showed a significantly positive correlation with MBL, TML and Nba in adult. For MBL and TML in preadolescence, but no significant correlation showed in 
adolescence, with a significant correlation for MSD with nearly all the related craniofacial dimensions in all age group in general. In all measurement the percentage of increase from preadolescence to adolescence more than that from adolescence to adult these were listed in Tables $(12,13)$.

Table (1): Descriptive statistic for preadolescent group.

\begin{tabular}{ccccc}
\hline Variables & Minimum/ V & Maximum/ V & Mean & $\begin{array}{c}\text { Standard } \\
\text { deviation }\end{array}$ \\
\hline MSH & 19.77 & 28.21 & 25.38 & 2.25 \\
MSD & 27.00 & 34.87 & 31.50 & 2.31 \\
SN & 59.87 & 68.27 & 63.32 & 2.15 \\
SBa & 37.91 & 48.80 & 43.62 & 3.19 \\
NBa & 94.00 & 102.67 & 98.13 & 2.74 \\
UAFH & 43.00 & 46.34 & 44.79 & 1.12 \\
LAFH & 51.13 & 61.05 & 55.65 & 2.68 \\
TAFH & 91.50 & 102.00 & 97.71 & 3.05 \\
UPFH & 34.25 & 40.14 & 36.86 & 1.93 \\
LPFH & 28.58 & 34.70 & 32.06 & 2.12 \\
TPFH & 56.82 & 63.90 & 60.20 & 2.07 \\
MaxL & 43.31 & 46.39 & 44.69 & 1.05 \\
MRL & 41.73 & 50.86 & 45.49 & 3.03 \\
MBL & 51.89 & 60.10 & 56.39 & 2.73 \\
TML & 85.70 & 94.70 & 90.04 & 3.02 \\
\hline
\end{tabular}

Number of group (14).

Table (2): Descriptive statistic for adolescent group.

\begin{tabular}{ccccc}
\hline Variables & Minimum/ V & Maximum/ V & Mean & Standard deviation \\
\hline MSH & 24.89 & 41.00 & 29.91 & 2.93 \\
MSD & 24.40 & 42.07 & 34.30 & 3.15 \\
SN & 55.67 & 71.10 & 65.85 & 3.09 \\
SBa & 40.61 & 54.34 & 45.74 & 2.78 \\
NBa & 94.29 & 109.00 & 101.39 & 3.40 \\
UAFH & 42.87 & 59.58 & 48.81 & 3.55 \\
LAFH & 52.99 & 69.49 & 61.10 & 3.08 \\
TAFH & 93.95 & 120.03 & 107.45 & 5.55 \\
UPFH & 34.78 & 48.11 & 41.67 & 2.67 \\
LPFH & 27.99 & 51.09 & 37.31 & 3.60 \\
TPFH & 58.87 & 82.33 & 67.97 & 4.86 \\
MaxL & 41.29 & 54.67 & 46.95 & 3.03 \\
MRL & 44.08 & 58.59 & 50.39 & 3.68 \\
MBL & 53.17 & 74.00 & 63.10 & 4.44 \\
TML & 90.21 & 113.48 & 100.59 & 4.24 \\
\hline
\end{tabular}

Number of group (70). 
Table (3): Descriptive statistic for adult group.

\begin{tabular}{ccccc}
\hline Variables & Minimum/ V & Maximum/ V & Mean & Standard deviation \\
\hline MSH & 27.70 & 46.69 & 32.94 & 4.39 \\
MSD & 28.39 & 40.80 & 53.09 & 3.86 \\
SN & 61.88 & 70.93 & 66.06 & 2.42 \\
SBa & 39.00 & 52.83 & 45.93 & 3.74 \\
NBa & 93.42 & 110.04 & 100.21 & 3.81 \\
UAFH & 42.58 & 53.28 & 47.99 & 2.69 \\
LAFH & 55.71 & 83.44 & 67.96 & 6.10 \\
TAFH & 102.48 & 135.84 & 113.50 & 6.60 \\
UPFH & 38.51 & 51.34 & 43.29 & 3.22 \\
LPFH & 30.35 & 48.18 & 41.05 & 4.69 \\
TPFH & 60.29 & 84.23 & 70.73 & 6.62 \\
MaxL & 40.22 & 53.64 & 48.53 & 3.25 \\
MRL & 42.85 & 65.52 & 54.96 & 4.49 \\
MBL & 58.51 & 73.98 & 67.53 & 4.22 \\
TML & 95.94 & 121.22 & 109.48 & 6.12 \\
\hline
\end{tabular}

Number of group (29).

Table (4): Correlation between MSH and craniofacial dimensions in preadolescent group.

\begin{tabular}{ccccccccccc}
\hline UAFH & LAFH & TAFH & \multicolumn{2}{c}{ UPFH } & LPFH & TPFH & SBa & NBa & MRL & TML \\
\hline$p$-value & 0.904 & 0.168 & 0.531 & 0.345 & 0.588 & $0.001^{*}$ & 0.088 & 0.362 & $0.020^{*}$ & 0.841 \\
\hline
\end{tabular}

*means a significant correlation.

Table (5): Correlation between MSH and craniofacial dimensions in adolescent group.

\begin{tabular}{lllllllllll}
\hline & UAFH & LAFH & TAFH & UPFH & LPFH & TPFH & SBa & NBa & MRL & TML \\
\hline$p$-value & $0.002^{*}$ & $0.004^{*}$ & $0.000^{*}$ & $0.000^{*}$ & 0.942 & $0.004^{*}$ & $0.003^{*}$ & $0.002^{*}$ & $0.035^{*}$ & $0.042^{*}$ \\
\hline *means a significant correlation
\end{tabular}

Table (6): Correlation between MSH and craniofacial dimensions in adult group.

\begin{tabular}{lcccccccccc}
\hline & UAFH & LAFH & TAFH & UPFH & LPFH & TPFH & SBa & NBa & MRL & TML \\
\hline$p$-value & $0.000^{*}$ & $0.000^{*}$ & $0.000^{*}$ & $0.000^{*}$ & 0.569 & 0.080 & $0.000^{*}$ & $0.000^{*}$ & 0.0 .204 & $0.003^{*}$ \\
\hline$*$ means a significant correlation.
\end{tabular}


Table (7): Correlation between MSH and craniofacial dimensions with the groups in general.

\begin{tabular}{|c|c|c|c|c|c|c|c|c|c|c|}
\hline & UAFH & LAFH & TAFH & UPFH & LPFH & TPFH & SBa & NBa & MRL & TML \\
\hline $\begin{array}{c}p- \\
\text { value }\end{array}$ & $0.000 *$ & $0.000^{*}$ & $0.000 *$ & $0.000 *$ & $0.000 *$ & $0.000^{*}$ & $0.000 *$ & $0.000 *$ & $0.000 *$ & $0.000 *$ \\
\hline
\end{tabular}

Table (8): Correlation between MSD and craniofacial dimensions in Preadolescent group.

\begin{tabular}{lccccc}
\hline & SN & NBa & MaxL & MBL & TML \\
\hline$p$-value & 0.336 & 0.088 & 0.051 & $0.016^{*}$ & $0.014^{*}$ \\
\hline *means a significant correlation.
\end{tabular}

Table (9): Correlation between MSD and craniofacial dimensions in adolescent group.

\begin{tabular}{lccccc}
\hline & SN & NBa & MaxL & MBL & TML \\
\hline$p$-value & 0.410 & 0.967 & 0.997 & 0.597 & 0.741 \\
\hline *means a significant correlation.
\end{tabular}

Table (10): Correlation between MSD and craniofacial dimensions in adult group.

\begin{tabular}{lccccc}
\hline & SN & NBa & MaxL & MBL & TML \\
\hline$p$-value & 0.498 & $0.009 *$ & 0.403 & $0.002 *$ & $0.023^{*}$ \\
\hline *means a significant correlation.
\end{tabular}

Table (11): Correlation between MSD and craniofacial dimensions with the age group in general.

\begin{tabular}{clllll}
\hline & SN & NBa & MaxL & MBL & TML \\
\hline$p$-value & $0.038^{*}$ & $0.010^{*}$ & 0.074 & $0.001 *$ & $0.000^{*}$ \\
\hline \multicolumn{6}{c}{ *means a significant correlation }
\end{tabular}


Table (12): The percent of increase in measurement from preadolescent to adolescent group.

\begin{tabular}{cc}
\hline Variables & Percent of increase \\
\hline MSH & $17.80 \%$ \\
MSD & $8.89 \%$ \\
SN & $3.98 \%$ \\
SBa & $4.86 \%$ \\
NBa & $3.33 \%$ \\
UAFH & $9.13 \%$ \\
LAFH & $9.79 \%$ \\
TAFH & $9.97 \%$ \\
UPFH & $13.05 \%$ \\
LPFH & $16.34 \%$ \\
TPFH & $12.89 \%$ \\
MaxL & $5.06 \%$ \\
MRL & $10.75 \%$ \\
MBL & $11.92 \%$ \\
TML & $11.72 \%$ \\
\hline
\end{tabular}

Table (13): The percent of increase in measurement from adolescent to adult group.

\begin{tabular}{cc}
\hline Variables & Percent of increase \\
\hline MSH & $10.16 \%$ \\
MSD & $2.30 \%$ \\
SN & $0.32 \%$ \\
SBa & $0.42 \%$ \\
NBa & $1.16 \%$ \\
UAFH & $1.68 \%$ \\
LAFH & $11.21 \%$ \\
TAFH & $5.63 \%$ \\
UPFH & $3.89 \%$ \\
LPFH & $10.02 \%$ \\
TPFH & $4.08 \%$ \\
MaxL & $3.34 \%$ \\
MRL & $9.07 \%$ \\
MBL & $7.00 \%$ \\
TML & $8.84 \%$ \\
\hline
\end{tabular}

Sinus growth or enlargement probably exert forces (functional matrix) on the surrounding structures that may stimulate its growth by bone remodeling and sutural growth process. This progressive sequential movement of component parts as a bone enlarge is termed relocation which is considered as a base of remodeling. "bone" doesn't regulate its own growth, the genetic and epigenetic determinants of skeletal development are in the functional tissue matrix. Indeed, the functional factors are the very agent that cause the bone to develop into its definite shape and size and to occupy the location it does. ${ }^{(13,14)}$

Enlow ${ }^{(15)}$ identifies the maxillary tuberosity as being a major site of maxillary growth, it does not, however, provide for the growth of the whole maxilla, but rather is responsible for the lengthening of the maxillary arches, probably this may explain the results that no correlation between the MSD and the MaxL.

From the data we notice that sinus dimensions showed a significant and positive correlation with the facial dimensions (maxillary height and mandibular length), this is probably may be due to the close relation between the sinus and the maxillary structure and the mandibule may be indirectly affected by the sinus, by the fact, that add to the process of bone apposition and resorption, the mandibule affected by the maxillary growth (secondary displacement), this is, with influence come from the cranial base through the temporomandibular joint.

For the cranial base it showed a little correlation when compared with facial structures this is could be explained by a fact that the cranial base away from the sinus and it is closely related to the brain and the brain growth play a fundamental role as functional matrix in cranial base growth.

In all measurement the percentage of increase from preadolescent to adolescent more than from adolescent to adult this could be postulated by the fact that growth start with acceleration from birth to adolescent period that reach to nearly a steady stage then followed by 
acceleration at adolescences reaching to the peak level at this period then the face gating its usual final position at beginning of adult period ${ }^{(5,15)}$ the result of this study also may be used in conjunction with this postulation.

Facial growth characterized by continuous and time-dependent rates of changes (i.e. skull sizes are different at different ages). Facial growth continues throughout life. Rate of change decreases over time. The anterior cranial base is more closely related to the maxilla because the nasomaxillary complex is suspended from the anterior cranial base. The articulation of the mandible (glenoid fossa) is located in posterior cranial base. The major determinant of growth in the maxilla is the enlargement of the nasal and oral cavities, including the sinuses which grow in response to functional needs. This is called the orofacial capsular matrix ${ }^{(4,5)}$.

$\operatorname{Moss}^{(7)}$ related the flexion of the cranial base angle to the brain growth and indirectly to the remodeling taking place in the cribiform plate. This probably could explain the little correlation of maxillary sinus with the cranial base which were represented in the tables add to the low percentage of increase in the cranial base dimensions from age group to another when compared to high percent of increase in the sinus dimensions.

\section{CONCLUSIONS}

Finally we could concluded that in the three age groups the growth of the maxillary sinus probably may influence the related craniofacial structures as a functional matrix role in the growth mechanism.

\section{REFERENCES}

1. Hauman CHJ, Chandler NP, Tong DC. Endodontic implications of the maxillary sinus: A review. Intern endod J. 2002; 35: 127-141.

2. Perrella A, Rocha SD, CavalcantiI MD. Quantitative analysis of maxillary sinus using computed tomography. $J$ Appl Oral Sci. 2003; 11(3): 229-33.

3. Mateus AR, Dolci JE, Costa HO, Sousa FC, Biase ND. Experimental study on the influence of facial muscle activity on the facial mesostructure bones in rabbits. Rev Bras Otorrinolaringol. 2008; 74(5):685-90

4. Moss ML. The primacy of functional matrices in orofacial growth . Dental Practitioner and Dental Record. 1968; 19: $65-73$.

5. Moss ML. The differential roles of periosteal and capsular functional matrices in orofacial growth. Europ $J$ Orthod. 2007; $29: 96-101$.

6. Enlow DH. Handbook of Facial Growth. $3^{\text {rd }}$ ed. Philadelphia W.B. Saunders Company. 1990.

7. Moss ML. Growth and development of the craniofacial complex: An epigenetic view point. New York, Thieme Medical Publishers. 1995.

8. Ballantyne JC. A ssynopsis of otolaryngology. $3^{\text {th }}$ ed. Varghese, Mumbai. 1978; Pp: 161-162.

9. Weiglein A. Radiologic anatomy of the paranasal sinuses in the children. Surg Radiol Anat. 1992; 14: 335-9.

10. Lund VJ. The maxillary sinus in the higher primates. Arch Otolaryngol. 1988; 105: 163-71.

11. Farah G, Nafis A. Morphometric analysis of developing maxillary sinuses in human foetuses. Int J Morphol. 2006; 24(3): 303-308.

12. Al-Sultan MM. Cranial base parameters effect on the nasomaxillary in Class II division 1 malocclusion (Three dimensional cephalometric study). MSc. Thesis. College of Dentistry. University of Mosul. 2005.

13. Moss ML. The primary role of functional matrices in facial growth. Am J Orthod. 1969; 55: 566-77.

14. Hans, Enlow. Essentials of facial growth. Textbook. Mosby. 1996.

15. Enlow DH. Handbook of facial growth.1982; Pp: 66-100. 